Katarzyna Buszko ${ }^{1 *}$, Karolina Obońska ${ }^{2 *}$, Piotr Michalski ${ }^{3}$, Agata Kosobucka ${ }^{3}$, Aleksandra Jurek ${ }^{2}$, Marzena Wawrzyniak ${ }^{2}$, Wioleta Stolarek ${ }^{2}$, Łukasz Pietrzykowski ${ }^{3}$, Anna Andruszkiewicz ${ }^{3}$, Aldona Kubica ${ }^{3}$

${ }^{1}$ Department of Theoretical Foundations of Biomedical Science and Medical Informatics, Collegium Medicum,

Nicolaus Copernicus University, Bydgoszcz, Poland

2Department of Cardiology and Internal Medicine, Collegium Medicum, Nicolaus Copernicus University, Bydgoszcz, Poland

${ }^{3}$ Department of Health Promotion, Collegium Medicum, Nicolaus Copernicus University, Bydgoszcz, Poland

*Katarzyna Buszko and Karolina Obońska have equally contributed to the present paper and should be considered to be the first authors

\title{
The Adherence Scale in Chronic Diseases (ASCD). The power of knowledge: the key to successful patient - health care provider cooperation
}

\author{
Corresponding author: \\ Katarzyna Buszko, PhD \\ Department of Theoretical \\ Foundations of Biomedical Science \\ and Medical Informatics, \\ Collegium Medicum, \\ Nicolaus Copernicus University, \\ Jagiellońska St. 13, \\ 85-067 Bydgoszcz, Poland \\ e-mail:buszko@cm.umk.pl
}

Medical Research Journal 2016; Volume 1, Number 1, 37-42 10.5603/MRJ.2016.0006 Copyright (C) 2016 Via Medica ISSN 2451-2591

\begin{abstract}
Introduction. Patients' adherence to long-term therapies is low. It translates into reduced quality of life and significant deterioration of health economics. Identification of potential barriers of medication-related adherence is a starting point allowing implementation of more advanced interventions directed to adherence improvement.

Aim. The purpose of our study was to create and validate a simple instrument used to assess patients' adherence to recommended medications.

Material and methods. The Adherence Scale in Chronic Diseases is a self-reported questionnaire with 8 items and with proposed 5 sets of answers. The total score in the Adherence Scale in Chronic Diseases ranges from 0 to 32 points. Three levels of adherence were considered (low: scores of 0 to 20; medium 21 to 25; high > 26). The validation of the questionnaire was conducted in accordance with the validation procedure. Assessment of the internal consistency was performed using $\alpha$-Cronbach coefficient. In order to conduct the factor analysis, we assessed: the determinant of correlation matrix, Kaiser-Mayer-Olkin (K-M-O) statistic and the Bartlett's test of sphericity. Factor analysis was conducted using principal component analysis with Oblimin rotation. The Kaiser criterion and scree plot were used in order to determine components of the questionnaire. Adherence levels were determined based on the percentiles.

Results. Grand total of 413 patients with a cardiovascular disease were included in the study. The reliability and homogeneity of the questionnaire were confirmed by $\alpha$-Cronbach coefficient (0.739). Factor analysis showed that in this questionnaire we can extract two components. The analysis of factor loadings indicated excluding item 2 from the questionnaire. After exclusion of the mentioned item, we repeated the validation procedure. For such a new dataset, according to the Kaiser criterion, only one component was extracted. Conclusions. The Adherence Scale in Chronic Diseases is a practical, reliable, consistent and well validated instrument for identifying specific obstacles to medication adherence. Its simplicity causes that it can be successfully applied in daily practice by health care professionals. Our survey has the potential to improve patient — health care professional communication and relationship.

Key words: adherence, barriers of adherence, compliance, medication, self-report scale, patient education, persistent therapy
\end{abstract}

Med Res J 2016; 1 (1): 37-42

\section{Introduction}

Over the last centuries, there was a remarkable progress achieved in medicine and pharmacology. The discovery of antibiotics, antivirals, vaccines, antihyper- tensive drugs, antiplatelet and antithrombotic medications, new surgical techniques as well as percutaneous methods of invasive treatment of many diseases have led to a significant improvement in survival of people. However, current morbidity and mortality rates do not 
allow us to celebrate a complete success. In everyday practice health care professionals face a dilemma: why, despite our effort, so many patients do not respond to provided therapies appropriately? Sometimes we tailor the therapy accordingly to an individual patient to achieve the best result, but even then instead of reward we get frustration. Implementation of new methods and therapeutic strategies is extremely important, but every time we have to take a step backwards we are obliged to look closer at our relation with a patient. Does the patient know why we recommend a specific therapy? Why is it so important to follow it consistently? Does the patient realize the consequences of non-adherence? Sometimes non-adherence to therapies might not only be dangerous but even lethal.

Starting from Hippocrates [1] doctors pay special attention to patients who are at risk of not following their recommendations. A phenomenon describing the above mentioned situation is called adherence. Throughout the years, the definition of adherence evolved [2-4]. Currently adherence to medications is defined as a process by which patients take their medications as prescribed by health care professionals [2]. There are three components of adherence: initiation, implementation and discontinuation. Initiation is defined as the starting point of the therapy - when a patient takes the first dose of a prescribed medication. Implementation is the extent to which patient's actual dosing corresponds to the prescribed dosing regimen, from initiation until the last dose, whereas the discontinuation occurs when a patient stops taking the prescribed medication, for whatever reason(s) [2].

In the literature, there are many determinants defined as responsible for non-adherence [5-7]. In general, they can be grouped into following sets: socio-economic-related factors, healthcare team- and system-related factors, condition-related factors, therapy-related factors, and patient-related factors [7]. Nevertheless, still many patients escape our attention imperceptibly. In developed countries adherence to long term therapy for chronic illnesses averages $50 \%$, while in developing countries the rates are even lower [8]. Poor adherence to long-term therapies reduces the effectiveness of treatment making it a critical issue in population health in terms of quality of life and of health economics [8]. Interventions aimed at improving adherence should provide a significant positive return on investment through primary prevention and secondary prevention of adverse health outcomes [8].

Hence the need for an instrument allowing us to identify immediately the group of patients potentially endangered to be non-adherent to the ordered therapy. The tool must be as simple as possible because complex and time consuming instruments will not be applied in everyday practice, simply because of the limited time for the patient during the stay in the clinic.

The purpose of our study was to create and validate a simple instrument used to assess patients' adherence to recommended medications. Furthermore, thanks to a proper set of questions in the survey we want to determine the most common reasons of non-adherence.

\section{Material and methods}

\section{Study group}

The study was conducted in the Cardiology Department of the Antoni Jurasz University Hospital No. 1 in Bydgoszcz and in the Cardiology Outpatient Clinic of the Antoni Jurasz University Hospital in Bydgoszcz from May 2015 to December 2015. The study was approved by the Local Bioethical Committee of the Nicolaus Copernicus University, Ludwik Rydygier Collegium Medicum in Bydgoszcz (study approval reference number KB 478/2015).

\section{Survey}

The Adherence Scale in Chronic Diseases was designed to facilitate identification of mechanisms determining adherence in adult patients with chronic illnesses as well as reflect the actual implementation of the treatment plan in terms of provided pharmacotherapy. It is a self-reported questionnaire with 8 questions and with proposed 5 sets of answers. All the questions refer to determinants of adherence associated with behavior and determinants that can indirectly influence the adherence and are related to situations and convictions (Tab. 1). The total score on the Adherence Scale in Chronic Diseases can range from 0 to 32 points, with scores $<24,24$ to 28 and $>29$ reflecting low, medium and high adherence respectively.

\section{Statistical analysis}

Statistical analyses were conducted using IBM SPSS Statistic version 23.0. All statistical tests were performed on the significance level $\alpha=0.05$. The validation of the survey was conducted in accordance with the validation procedure. We assessed the internal consistency using $\alpha$-Cronbach coefficient. In order to conduct the factor analysis, we assessed: the determinant of correlation matrix, Kaiser-Mayer-Olkin (K-M-O) statistic and the Bartlett's test of sphericity. According to the literature, the sample size of 413 responders can produce correct fractional structure [9]. Factor analysis was conducted using principal component analysis with Oblimin rotation. The Kaiser criterion and scree plot were used in order to determine components of the questionnaire. 
Table 1. The Adherence Scale in Chronic Diseases

1. Do you always remember to take all your medications according to your doctor's instructions?
A. Always.
B. Almost always.
C. Sometimes.
D. Hardly ever.
E. Never.

2. Do you find purchasing of the medications prescribed by your doctor a significant financial burden?
A. No, it is insignificant.
B. It is burdensome, but acceptable.
C. It is financially cumbersome, but with some effort I can still afford to buy all my medications.
D. Occasionally I cannot afford to buy all my medications.
E. Frequently I cannot afford to buy all my medications.

3. Do you happen to change the dosing of your medications without prior consultation with your doctor?
A. Never.
B. Only occasionally.
C. Sometimes.
D. Frequently.
E. I do not adhere to my doctor's recommendations at all.

4. Do you adjust the dosing of your medications according to how you feel?
A. No, I strictly follow the prescribed dosing, no matter how I feel.
B. Yes, I reduce the dosage of some medications when I feel good.
C. Yes, I skip doses of some medications when I feel good.
D. Yes, I temporarily discontinue some medications when I feel good.
E. Yes, I discontinue all medications when I feel good.

5. On the appearance of medication-related side effects (e.g. stomach pain, liver pain, rash, lack of appetite, oedema):
A. I seek medical attention instantly.
B. I reduce the dosage of the medication and attempt to expedite the elective appointment with my doctor.
C. I discontinue the medication and attempt to expedite the elective appointment with my doctor.
D. I discontinue the medication and wait for the next elective appointment with my doctor.
E. I discontinue all my medications and wait for the next elective appointment with my doctor.

6. Do you find all your medications necessary for your health?
A. Yes, I do.
A. Yes, on every appointment.
B. Yes, he/she usually does.
C. Yes, but only sometimes.
D. Yes, but only occasionally.
E. No, never.

B. I find most of my medications to be beneficial for my health.

C. I find only some of my medications to be beneficial for my health.

D. I find some of my medications to be beneficial for my health, while the others to be harmful for me.

E. I find the majority of my long-term medications to be harmful for me.

7. Does your doctor inquire about medication-related problems that you might possibly experience?

8. Do you tell truth when asked by your doctor about medication-related problems?
A. Yes, always.
B. Almost always.
C. I try to be honest, but sometimes it is hard to admit to non-compliance with doctor's recommendations
D. Sometimes yes, another time no.
E. No, I don't. I find it my own private business.

We also determined the adherence levels based on the percentiles.

\section{Results}

Four hundred and thirteen patients with a cardiovascular disease were included into the study. The mean age was $60.8 \pm 9.7$ years, and $27.1 \%$ of the total number (112 of 413) were women. The questionnaires were completed anonymously by 401 patients.

\section{Validation of the Adherence Scale in Chronic Diseases}

The best known tool used to check the internal consistency of a questionnaire is the $\alpha$ - Cronbach coefficient. Therefore, we assessed the internal consistency 
Table. 2. Correlation coefficients for all 8 items

\begin{tabular}{lcccccccc}
\hline Item & $\mathbf{1}$ & $\mathbf{2}$ & $\mathbf{3}$ & $\mathbf{4}$ & $\mathbf{5}$ & $\mathbf{6}$ & $\mathbf{7}$ \\
\hline 1 & $\mathbf{1 . 0 0 0}$ & 0.112 & 0.412 & 0.391 & 0.311 & 0.263 & 0.162 \\
2 & 0.112 & $\mathbf{1 . 0 0 0}$ & 0.163 & 0.102 & 0.182 & 0.230 & 0.129 \\
3 & 0.412 & 0.163 & $\mathbf{1 . 0 0 0}$ & 0.539 & 0.404 & 0.298 & 0.266 \\
4 & 0.391 & 0.102 & 0.539 & $\mathbf{1 . 0 0 0}$ & 0.401 & 0.346 & 0.212 \\
5 & 0.311 & 0.182 & 0.404 & 0.401 & 1.000 & 0.358 & 0.281 \\
6 & 0.263 & 0.230 & 0.298 & 0.346 & 0.358 & 1.000 & 0.343 \\
7 & 0.162 & 0.129 & 0.266 & 0.212 & 0.281 & 0.343 & 1.000 \\
8 & 0.356 & 0.084 & 0.445 & 0.425 & 0.337 & 0.289 & 0.218
\end{tabular}

using $\alpha$-Cronbach coefficient and we obtained the value 0.739 . According to the literature, such result confirmed reliability and homogeneity of the questionnaire [10]. We also calculated the $\alpha$-Cronbach coefficient for the questionnaire after removing individual questions. The $\alpha$-Cronbach coefficient was higher (0.752) when item 2 was not used for computation. The coefficient was also slightly higher (0.746) when item 7 was excluded from computations. We estimated the correlation coefficients between individual items. The analysis showed, that item 2 has the lowest correlations with other items. The highest correlation coefficient for this item was 0.23 (correlation coefficient with item 6). All correlation coefficients are presented in Table 2.

In the procedure of validation one must verify the validity structure and indicate the dimension of questionnaire. The presence of multidimensionality is connected with the presence of highly correlated group of items. We also analyzed the scale in this respect using factor analysis. This analysis can generate the possible subscales which are represented by sets of items within the questionnaire. At first we exanimated the assumption of factor analysis for the analyzed data. We also assessed: the determinant of correlation matrix, Kaiser-Mayer-Olkin (K-M-O) statistic (to measure sampling adequacy) and we conducted the Bartlett's test of sphericity. The determinant of correlation matrix was 0.196 , the value of K-M-O statistic was 0.847 and the Bartlett's test of sphericity was statistically significant. Such results indicated the eligibility of the dataset for using factor analysis. The Shapiro-Wilk test was not statistically significant, therefore, the factor analysis was conducted using principal component analysis with Oblimin rotation. We determined the components of the questionnaire in line with the Kaiser criterion. We obtained two components which accounted for about $52 \%$ of variance in the dataset. Figure 1 shows the scree plot with marked eigenvalues for each factor. The scree plot confirmed the choice of the two components.

Table 3 presents the factor loadings for each items. One can determine the items which create those

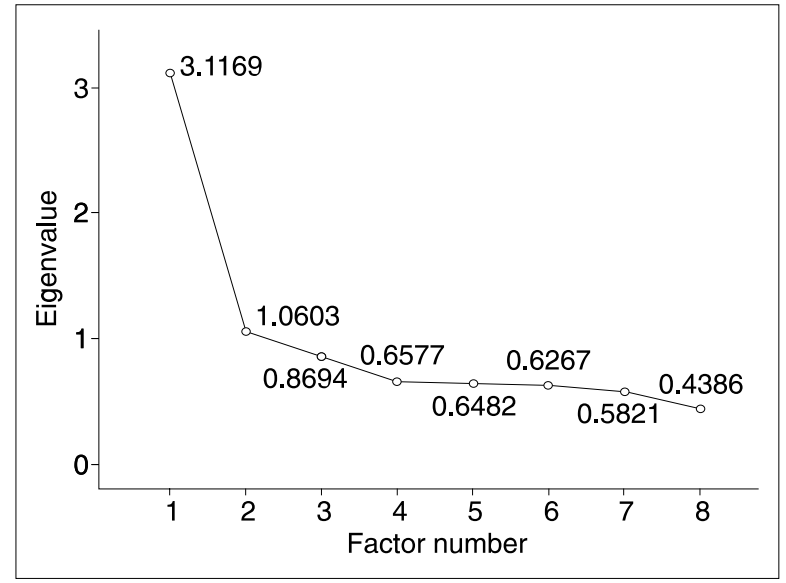

Figure 1. Scree plot

Table 3. The values of factors loading for the items 1-8

\begin{tabular}{lcc}
\hline Item & Component 1 & Component 2 \\
\hline 1 & 0.562 & -0.004 \\
2 & $-\mathbf{0 . 0 3 2}$ & $\mathbf{0 . 3 3 9}$ \\
3 & 0.755 & -0.022 \\
4 & 0.722 & -0.012 \\
5 & 0.364 & $\mathbf{0 . 3 0 2}$ \\
6 & 0.025 & 0.679 \\
7 & 0.048 & 0.452 \\
8 & 0.600 & 0.011 \\
\hline
\end{tabular}

two components. It is recognized that criteria for such determination are arbitrary. In the literature it is suggested that if the value of loading factor is less than 0.4 , it indicates low factor loadings. In the case of our dataset, for the first component, items: 1, 3, 4 and 8 had factors loading $>0.4$, while for the second component, items 6 and 7 showed factors loading $>0.4$. Items 2 and 5 did not have sufficient loading. We denoted the lowest factor loading for item 2 ( -0.032 for the first component and 0.339 for the second one). 
We verified the value of the correlation coefficient for those two components and it was 0.633 . Therefore, we concluded that the components are sufficiently correlated. In the light of such results we excluded item 2 from the questionnaire.

We also conducted the validation procedure again for items: 1, 3-8. The $\alpha$-Cronbach coefficient was higher (0.752), which indicated satisfactory internal consistency. We examined the assumption of factor analysis for the new dataset. The determinant of correlation matrix was 0.211 , the value of K-M-O statistic was 0.848 and the Bartlett's test of sphericity was statistically significant. We conducted the factor analysis again, using principal component analysis with Oblimin rotation. For the analyzed data, according to the Kaiser criterion, we could extract only one component.

The exclusion of item 2, which had the lowest correlation coefficient with other items, showed that in fact the proposed questionnaire focuses on measurement of one coherent target. Now an attempt to extract more than one component through factor analysis will fail, because even if such components exist, they are highly correlated.

\section{Adherence levels}

We described a new tool, which can measure the adherence. Every new questionnaire should be associated with a scale, which indicates the level of the measured effect. In the case of a scale for which the total score has normal distribution a sten score can be defined. In our dataset we could not do that because of the skewed distribution of the total score. Hence, we determined the adherence levels based on the percentile scale.

We assumed that the total score higher than 30 percentiles indicates low adherence, the total score between 30 and 70 percentiles indicates medium adherence and the total score higher than 70 percentiles indicates high adherence. The described criterion is presented in Table 4. In the table we included the adherence levels for the scale with maximum total score 32 and 28 (after excluding item 2).

We also present the descriptive statistics for the questionnaire. Table 5 contains the descriptive statistics for the original version of the questionnaire and the version without item 2. The adherence levels based on the percentile scale and the descriptive statistics reveal the effect of negatively skewed distribution of the total score. That is why the low level of adherence ends at 23 and the high score begins at 29. The median is 24 , and the minimum score is 9 .

\section{Discussion}

Our study demonstrated a high reliability and criterion-related validity of the Adherence Scale in Chronic
Table 4. Adherence levels

\begin{tabular}{lll}
\hline \multicolumn{2}{l}{ Maximum Total Score $=32$} & \\
\hline Percentile & Score & Level \\
\hline 10 & 20 & Low \\
20 & 23 & \\
30 & 24 & Medium \\
40 & 25 & \\
50 & 26 & \\
60 & 27 & \\
70 & 28 & High \\
80 & 29 & \\
90 & 31 & \\
\hline
\end{tabular}

Maximum Total Score $=\mathbf{2 8}$

\begin{tabular}{lll}
\hline Percentiles & Score & Level \\
\hline 10 & 18 & Low \\
20 & 20 & \\
30 & 21 & Medium \\
40 & 23 & \\
50 & 24 & \\
60 & 25 & \\
70 & 26 & High \\
80 & 27 & \\
\hline
\end{tabular}

Diseases. It is a simple and quick to carry out survey, which allows to assess the risk of non-adherence to medications used in chronic illnesses treatment. Non-adherence is determined by several factors [5-7]. Our survey helps in their identification, especially in identification of behaviours associated as well as determinants of adherence related to situations and convictions. The knowledge of high possibility of non-adherence to the recommended therapy by our patient motivates us to improve our engagement since some of our patients require more attention than the others. The effort put into our work with a patient is extremely important and, what is more, irreplaceable. It can prevent misunderstanding of the recommendation and makes our patients feel important members of the team engaged in the therapeutic process, instead of being passive recipients.

The presented questionnaire is accurate and consistent. However, the performed analyses have demonstrated that second question regarding financial aspects of the treatment lowers the consistency of the test and therefore needs to be modified or excluded. This item was the only one in the survey raising financial issues and its detachment from the other questions is not surprising. Moreover, financial aspect of the treatment is a very important issue in all populations [11] and it might have an impact on responders' answers. In our further studies we plan to modify this question appropriately, thereby increasing the consistency of the questionnaire. 
Table 5. Descriptive statistics for the questionnaire

\begin{tabular}{llllllll}
\hline Item & Mean & Std & Median & Min & Max & Q1 & Q3 \\
\hline 1 & 3.55 & 0.59 & 4.00 & 1.00 & 4.00 & 3.00 & 4.00 \\
2 & 2.67 & 0.86 & 3.00 & 0.00 & 4.00 & 2.00 & 3.00 \\
3 & 3.47 & 0.74 & 4.00 & 1.00 & 4.00 & 3.00 & 4.00 \\
4 & 3.60 & 0.73 & 4.00 & 0.00 & 4.00 & 3.00 & 4.00 \\
5 & 3.37 & 0.85 & 4.00 & 0.00 & 4.00 & 3.00 & 4.00 \\
6 & 3.31 & 0.85 & 3.00 & 0.00 & 4.00 & 3.00 & 4.00 \\
7 & 2.20 & 1.26 & 2.00 & 0.00 & 4.00 & 1.00 & 3.00 \\
8 & 3.55 & 0.72 & 4.00 & 0.00 & 4.00 & 3.00 & 4.00 \\
Total score & 25.72 & 4.04 & 26.00 & 11.00 & 32.00 & 23.00 & 29.00 \\
$(1-8)$ & & & & & & & 21.00 \\
Total score & 23.05 & 3.76 & 24.00 & 9.00 & 28.00 & & 26.00 \\
$(1.3-8)$ & & & & & & &
\end{tabular}

\section{Limitations of the study}

Several limitations of our study need to be acknowledged. The main limitation is that our study was conducted in a single medical centre. Further, the study population was small, and the survey must be conducted in a larger population of patients with chronic illnesses. The questionnaire was performed anonymously and in the future patient characteristic must be included in the analysis. Moreover, we did not compare our survey results with an objective method of adherence measurement such as: National Health Found reports, electronic monitoring, drug concentration in blood, etc. The survey was not compared with any other questionnaires. In the future studies, comparison of the Adherence Scale in Chronic Diseases with the most often used 8-item Morisky Medication Adherence Scale should be included.

\section{Conclusion}

The Adherence Scale in Chronic Diseases is a practical, reliable, consistent and well validated instrument for identifying specific obstacles to medication adherence. Its simplicity causes that it can be successfully applied in daily practice by health care professionals. Our survey has the potential to improve patient - health care professional communication and relationship, which are the key points providing higher adherence to the specific therapy. Future studies are required to assess the actual connec- tion between the Adherence in Chronic Diseases Scale and the adherence measured with objective methods.

\section{References}

1. Lerner $\mathrm{BH}$. From careless consumptives to recalcitrant patients: the historical construction of noncompliance. Soc Sci Med 1997; 45: 1423-1431.

2. Vrijens B, De Geest S, Hughes DA et al.; ABC Project Team. A new taxonomy for describing and defining adherence to medications. $\mathrm{Br} \mathrm{J}$ Clin Pharmacol 2012; 73: 691-705.

3. Cramer JA, Roy A, Burrell A, Fairchild CJ, Fuldeore MJ, Ollendorf DA, Wong PK. Medication compliance and persistence: terminology and definitions. Value Health 2008; 11: 44-47.

4. Raebel MA, Schmittdiel J, Karter AJ, Konieczny JL, Steiner JF. Standardizing terminology and definitions of medication adherence and persistence in research employing electronic databases. Med Care 2013: 51 (8 suppl. 3): S11-S21.

5. Kubica A, Obońska K, Fabiszak T, Kubica J. Adherence to antiplatelet treatment with P2Y12 receptor inhibitors. Is there anything we can do to improve it? A systematic review of randomized trials. Curr Med Res Opin 2016; 1-11 [Epub ahead of print].

6. Zeber JE, Manias E, Williams AF et al.; ISPOR Medication Adherence Good Research Practices Working Group. A systematic literature review of psychosocial and behavioral factors associated with initial medication adherence: a report of the ISPOR medication adherence \& persistence special interest group. Value Health 2013; 16: 891-900.

7. Kardas P. Lewek P, Matyjaszczyk M. Determinants of patient adherence: a review of systematic reviews. Front Pharmacol 2013; 4: 91.

8. Sabate E. Adherence to Long-Term Therapies: Evidence for Action. Geneva: World Health Organization, 2003. Available from: http://www. who.int/chronic conditions/en/adherence report.pdf.

9. Costello AB, Osborne JW. Best Practices in Exploratory Factor Analysis: Four Recommendations for Getting the Most from Your Analysis. Practical Assessment, Research \& Evaluation 2005; 10, 7.

10. Rowe P. Essential Statistics for the Pharmaceutical Sciences. John Wiley \& Sons, UK, West Sussex 2016

11. Hennessy D, Sanmartin C, Ronksley P, Weaver R, Campbell D, Manns B, Tonelli M, Hemmelgarn B. Out-of-pocket spending on drugs and pharmaceutical products and cost-related prescription non-adherence among Canadians with chronic disease. Health Rep 2016; 27: 3-8 